Int. J. Dev. Biol. 54: 905-911 (2010)

doi: $10.1387 /$ ijdb.092882ql

\title{
Expression of protocadherin-19 in the nervous system of the embryonic zebrafish
}

\author{
QIN LIU*,1, YUN CHEN ${ }^{1}$, FUMITAKA KUBOTA ${ }^{2}$, JEAN J. PAN ${ }^{1}$ and TOHRU MURAKAMI ${ }^{3}$ \\ ${ }^{1}$ Department of Biology, University of Akron, Akron, Ohio, USA, ${ }^{2}$ Gunma University Hospital, Maebashi, \\ Gunma, Japan and ${ }^{3}$ Department of Anatomy, Gunma University Graduate School of Medicine, Maebashi, \\ Gunma, Japan
}

\begin{abstract}
We have analyzed the expression pattern of protocadherin-19, a member of the $\delta 2-$ protocadherins, in the nervous system of developing zebrafish using in situ hybridization methods. mRNA encoding protocadherin-19 (Pcdh19) began to be expressed at about 12 hours post fertilization (hpf) showing a segmental expression pattern in the anterior $1 / 3$ of the neural keel, with strong expression in the presumptive forebrain, cerebellum/rhombomere 1 and rhombomere 4. Pcdh19 expression in the posterior neural keel was continuous and confined to the midline region. By 24 hpf, Pcdh 19 was expressed widely in the brain and spinal cord, with higher expression levels in the ventral telencephalon, dorsal and central thalamus, optic tectum, central tegmentum, cerebellum and dorsolateral regions of the hindbrain. As development proceeded, Pcdh19 expression domains became restricted to the dorsal and/or lateral regions of the central nervous system, and Pcdh19 expression was not detected in the spinal cord of two- and threeday old embryos. Pcdh 19 was also expressed by the eye primordium, developing retina, lens and otic vesicle. Similar to its expression in the brain, Pcdh19 expression in the eye and ear was also spatially and temporally regulated.
\end{abstract}

KEY WORDS: development, cell adhesion molecule, central nervous system, retina, otic vesicle

\section{Introduction}

Cell adhesion molecules cadherins play important roles in tissue and organ development, function and maintenance of adult structures (Yagi and Takeichi 2000; Gumbiner 2005; van Roy and Berx 2008). So far more than 100 cadherins have been identified, and they are grouped into several subfamilies, including classical cadherins, protocadherins, desmosomal cadherins and flamingo cadherins (Nollet et al. 2000). The protocadherins (Pcdhs) subfamily contain more members than any other cadherin subfamilies, and Pcdhs are divided into several groups, such as clustered Pcdhs: $\alpha$-, $\beta$-, and $\gamma$-Pcdhs, and non-clustered $\delta$-Pcdhs (Suzuki 1996; Frank and Kemler 2002; Noonan etal. 2004; Wu 2005). The $\delta$-Pcdhs are further divided into $\delta 1$-Pcdhs and $\delta 2-P c d h s$ based mainly on presence of several conserved motifs in the cytoplasmic domains, with the $\delta 1$-Pcdhs (e.g. Pcdh1, 7 and 9) having three conserved motifs CM1-CM3, whereas the $\delta 2-P c d h s$ (e.g. Pcdh10, 18 and 19) containing only two (CM1 and CM2) of the motifs (Redies et al. 2005; Vanhalst et al. 2005).
Results and Discussion

\section{Zebrafish Pcdh19}

Zebrafish Pcdh19encodes a protein with a putative hydrophobic signal sequence, extracellular region containing six repeats, transmembrane and cytoplasmic domains (Fig. 1). Phylogenetic analysis (Fig. 2) and alignment of several Pcdhs sequences (Fig. 3) show that the zebrafish Pcdh19 is most similar to human and mouse Pcdh19 at the amino acid level, less similar to Pcdh18, another $\delta 2-P c d h$, and even less similar to Pcdh1, a $\delta 1$-Pcdh (Figs. 2 and 3 A,B). Like other $\delta 2-P c d h s$, the zebrafish Pcdh 19 contains only two conserved motifs, CM1 and CM2, in the cytoplasmic domain (Fig. 3A). The zebrafish CM1 is identical to human and mouse $\mathrm{CM} 1$, while the zebrafish $\mathrm{CM} 2$ has a high degree of identity $(88.2 \%)$ with the human and mouse CM2 (Fig. 3A). As in other

Abbreviations used in this paper: hpf, hours post fertilization; Pcdh19, protocadherin-19 gene or mRNA; Pcdh19, protocadherin-19 protein; Pcdhs, protocadherins;

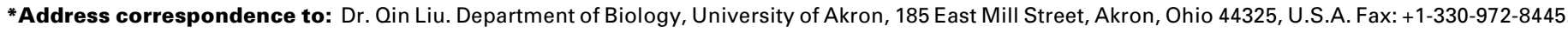
e-mail: qliu@uakron.edu
} 
Sig

MHSKDMDFVQMFVCELLCWTGVDAVFNLK

EC1

YTVEEELRAGTKIANVTADAKVAGFALGNRQPYLRVISNSEPRWVNLSPAGLLITKQKID RDAVCRQTPKCFISLEVMSNSMEICVIKIEIIDVNDNAPRF

\section{EC2}

PTNHIDIEISENAAPGTRFPLEGASDPDSGSNGIQTYTITPNDIFGLEIKTRGDGSKIAE LVVEKTLDRETQSRYTFELTAEDGGDPPKSGTVQLNIKVIDSNDNNPVF

EC3

DEPVYTVNVLENSPINTLVIDLNATDPDEGTNGEVVYSFINFVSNLTKQMFKIDPKTGVI TVNGVLDHEELHIHE I DVQAKDLGPNS I PAHCKVIVNVIDINDNAPE I

EC4

KLLSENSEMVEVSENAPLGYVIALVRVSDNDSGANGKVQCRLQGNVPFRLNEFESFSTLL VDGRLDREQRDMYNLTILAEDSGYPPLRSSKSFAVKVTDENDNPPYF

EC5

TKPHYQAMVLENNVPGAFLLAVSARDPDLGMNGTVSYEI IKSEVRGMSVESYVTVNSNGE IYGVRAFNHEDTRTEEFKVSAKDGGDPPLTSNATVRIVVLDVNDNTPVM

EC6

TTPPLVNGTAEVS I PKNAGVGYLVTQIKADDYDEGENGRLTYS ISEGDMAYFEIDQINGE VRTTKTFGENAKPSYQITVVAHDHGQTSLSASAYIVIYLSPDLNAQEQIGPVN TM

LSLIFIIALGSIAVILFVTMIFVAV

Cyto

KCKRDNKEIRTYNCRVAEYSYGNQKKSSKKKKLSKNDIRLVPRDVEETDKMNVVSCSSLT SS LNYFDYHQQTLPLGCRRSESTFLNVENQNSRNAAPNHGYHHTFTGQGPQQPDL I INGM PLPETENYSIDSSYVNSRAHLIKSTSTFKDMEGNSLKDSGHEESDOTDSEHDVORGHYAD TAVNDVLNMTVPSNNSQ I PDQDQSEGFHCQDECRI LGHSDRCWMPRVP I PARAKSPEHGR NVIALS IEATTVDVPHYEDCGTTKRTFATFGKDGPDEDRAEQRGRRQTAEPAVCSPKTNG AVREAGNGREAVSPITSPVHLKSPQSKAPSTYNTLKCRDAERIANHSLLRQPEGKDSEPA MREINTLLQDGRDKESPGSKRLKDIVI

Fig. 1. Deduced amino acid sequence of zebrafish Pcdh19. The putative hydrophobic signal sequence (Sig), and the conserved cytoplamic motifs 1 and 2 (CM1 and CM2, respectively) are underlined. Other abbreviations: cyto, cytoplasmic domain; EC1-EC6, extracellular domains 1-6; TM, transmembrane domain.

Pcdh domains, the zebrafish CM1 and CM2 are more similar to those of Pcdh18 ( $\delta 2)$ than Pcdh1 ( $\delta 1)$ (data not shown). Zebrafish Pcdh19 has two isoforms, which are identical except that isoform 1 is slightly shorter (missing amino acids 755-826 in the cytoplasmic domain, Fig. 1) than isoform 2. The human PCDH19 also has two variants but they differ by only one amino acid, with variant 1 containing an extra serine at amino acid 847 in the protein.

\section{Pcdh19 expression}

Compared to our extensive knowledge of classical cadherins (e.g. cadherin-1 and cadherin-2, also known as E-and N-cadherins, respectively) expression and function, little is known about Pcdh19 expression and function in developing vertebrates, and to the best of our knowledge, there is no published report on Pcdh19 expression in nonmammalian vertebrates.

Using reverse transcriptase-polymerase chain reaction (RTPCR) and whole mount in situhybridization methods, we analyzed expression of Pcdh19 in embryonic zebrafish from 6 hours post fertilization (hpf) to $72 \mathrm{hpf}$. RT-PCR experiments showed that both Pcdh19isoforms were expressed by embryos of $10 \mathrm{hpf}$ to $72 \mathrm{hpf}$, with isoform 2 expression slightly stronger than isoform 1 in 18-22 hpf and $72 \mathrm{hpf}$ embryos (Fig. 4A). cRNA probes designed to detect both pcdh19 isoforms were used to perform whole mount in situ hybridization. There was no Pcdh19 expression found in young embryos of $6 \mathrm{hpf}$ (Fig. 4B) and $9 \mathrm{hpf}$ (data not shown). At 12-13 hpf, Pcdh19expression was observed in the neural keel. In the anterior neural keel, Pcdh19expressing domains in the presumptive forebrain and hindbrain were separated by regions with little or no Pcdh19expression (Fig. 4 C,D). The eye primordia also contained Pcdh19(Fig. 4D). To determine the relative positions of the Pcdh19 expression domains in the presumptive hindbrain, we performed double-labeling experiments using digoxigenin-labeled Pcdh19cRNA probes coupled with fluoresceinlabeled pax2a (labeling the boundary of the mid- and hindbrains, Krauss et al. 1991) or krox20 (labeling the rhombomeres 3 and 5, Oxtoby and Jowett 1993). The first Pcdh19 expression domain (indicated by an arrow) in the presumptive hindbrain was located immediately posterior to the boundary between the mid-and hindbrains (Fig. 4F), while the second Pcdh19 expression domain (indicated by an arrowhead) in the presumptive hindbrain was located between the rhombomeres 3 and 5 labeled by the krox20 probe

563 (Fig. 4 G,H). Therefore, the first and second Pcdh19 expression domains in the presumptive hindbrain 676 were likely situated in the presumptive cerebellum/ 701 rhombomere 1 and rhombomere 4, respectively. Pcdh19 expression in the posterior neural keel was continuous, but appeared to be restricted to regions along the midline (indicated by two arrows in Fig. 4E).

At 18 hpf, Pcdh19was expressed in both the brain and spinal cord (Fig. 5 A-D), with obvious regional differences in expression levels, judged by staining intensities, in the fore- and midbrains (Fig. 5B). The ventral telencephalon, ventral diencephalon and tegmentum showed stronger Pcdh19 expression than the remaining regions of the fore- and midbrains. Similar to Pcdh19expression in the younger embryos (Fig. 4), reduced Pcdh19expression was found between the cerebellum and optic tectum (arrowhead in Fig. 5B). No regional difference in expression levels was detected in the hindbrain (Fig. 5C), except there was a narrow region, between the cerebellum and the remaining hindbrain, with reduced Pcdh19 expression (arrow in Fig. 5 B,C). This region was likely derived from the region between the first $P c d h 19$ expression domain and rhombomere 3 in the presumptive hindbrain of younger embryos (Fig. 4 G,H). In the spinal cord, Pcdh19expression appeared to be stronger in the floor plate region (Fig. 5D). In addition to the

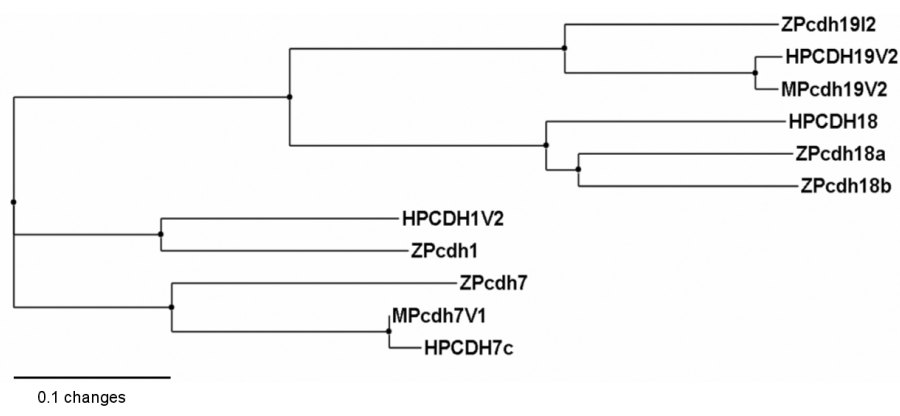

Fig. 2. Unrooted phylogram resulting from neighbor-joining distance analysis of EC1 through the carboxy-terminal protein sequence alignment. Abbreviations: human protocadherin-1 variant 2, HPCDH1V2; human protocadherin-7, variant C, HPCDH7c; human protocadherin-18, HPCDH18; human protocadherin-19 variant 2, HPCDH19V2; mouse protocadherin-7 variant 1, MPcdh7V1; mouse protocadherin-19 variant 2, MPcdh19V2; zebrafish protocadherin-1, ZPcdh1; zebrafish protocadherin-7, ZPcdh7; zebrafish protocadherin18a, ZPcdh18a; zebrafish protocadherin-18b, ZPcdh18b; zebrafish protocadherin-19 isoform 2, ZPcdh19/2. 
A ZPcdh19I2 HPCDH19V2 MPcdh19V2 HPCDH1 8 ZPcoh18a

ZPcdh19I2 HPCDH19V2 MPcdh19V2 HPCDH1 8

ZPCdh18a
1 YTVEEELRAGTKIANVTADAKVAGFALGNRQP-YLRVISNSEPRWVNLSP-AGLIITKQKIDRDAVCRQTPKCFISLEVMSN---SMEICVIKIEIID 1 YSVEEEQRAGTVIANVAKDAREAGEALDPROASAFRVVSNSAPHLVDINPSSGLIVTKOKIDRDLLCROSPKCIISLEVMSS---SMEICVIKVEIKD 1 YSVEEEQRAGTVIANVAKDAREAGFALDPRQASAFRVVSNSAPHLVDINPSSGLLVTKQKIDRDLLCRQSPKCI I SLEVMSS---SMEICVIKVEIKD 1 YRIYEEQRVGSVIARLSEDVADVLLKLPNPSTVRFRAMQRGNSPLIVVNEDNGEIS IGATI DREQLCQKNLNCS IEFDVITLPTEHLQLFHIEVEVLD 1 YQVYEEQKVGTVIARLREDVADVLSKLPSS I PLRFRAMQRGSASLLSVRDQDGEISIRTKI DREKLCEKNLNCTIEFDVLTLPTEHLQLFHIEVEILD

93 VNDNAPRFPTNHIDIEISENAAPGTRFPLEGASDPDSGSNGIQTYTITPNDIFGLEIKTRGDGSKIAELVVEKTLDRETQSRYTFELTAEDGGDPPKS 95 LNDNAPSFPAAQIELEISEAASPGTRI PLDSAYDPDSGSFGVQTYELTPNELFGLEIKTRGDGSRFAELVVEKSLDRETQSHYSFRITALDGGDPPRI 95 LNDNAPSFPAQIELEISEAASPGTRIPLDSAYDPDSGSFGVQTYELTPNELFGLEIKTRGDGSRFAELVVEKSLDRETQSHYSFRITALDGGDPPHM 98 INDNSPQFSRSLIPIEISESAAVGTRIPLDSAFDPDVGENSLHTYSLSANDFFNIEVRTRTDGAKYAELIVVRELDRELKSSYELQLTASDMGVPQRS 98 INDNAPQFARPVIPIEISETAAVGTRIPLDSATDPDVGENSLNTYSLTPSGFFKIDILTRTDGAKYAELVVLKELDREVRASYELQLTASDRGVPPKF

ZPcdh19I2 191 GTVQLNIKVIDSNDNNPVFDEPVYTVNVLENSPINTLVIDLNATDPDEGTNGEVVYSF INFVSNLTKQMFKIDPKTGVITVNGVLDHEELHIHEIDVQ HPCDH19V2 193 GTVGLSIKVTDSNDNNPVFSESTYAVSVPENSPPNTPVIRLNASDPDEGTNGQVVYSFYGYVNDRTRELFQIDPHSGLVTVTGALDYEEGHVYELDVQ MPcdh19V2 193 GTVGLSIKVTDSNDNNPVFGESTYSVSVPENSPPNTPVIRLNASDPDEGTNGQVVYSFYGYVNDRTRELFQIDPHSGLVTVTGALDYEEGHVYELDVQ HPCDH18 196 GSSILKISISDSNDNSPAFEQQSYI IQLLENSPVGTLLIDLNATDPDEGANGKIVYSFS SHVSPKIMETFKIDSERGHLTLFKQVDYEITKSYEIDVQ ZPcdh18a 196 GTTLLKISIADSNDNNPVFEKPSYVINLLENSPLGSLIIDLNATDPDEGTNGKIIYSFSSHVSPKILETFKINSDNGHLTLMRKVDFESTNSYDIDVQ

ZPcdh19I2 289 AKDLGPNSIPAHCKVIVNVIDINDNAP--EIKLLSE-NSEMVEVSENAPLGYVIALVRVSDNDSGANGKVQCRLQGNVPFRLN-EFESFSTLLVDGRI HPCDH19V2 291 AKDLGPNSIPAHCKVTVSVLDTNDNPP--VINLLSV-NSELVEVSESAPPGYVIALVRVSDRDSGLNGRVQCRLIGNVPFRLQ-EYESFSTILVDGRI MPcdh19V2 291 AKDLGPNSIPAHCKVTVSVLDTNDNPP--I INLLSV-NSELVEVSESAPPGYVIALVRVSDRDSGLNGRVQCRLLGNVPFRLQ-EYESFSTILVDGRI HPCDH18 294 AQDLGPNSI PAHCKII I KVVDVNDNKPE ININLMSPGKEE ISYIFEGDPIDTFVALVRVQDKDSGLNGE IVCKLHGHGHFKLQKTYENNYLILTNATL ZPcdh18a 294 AQDMGPNSMPAHCKVI IKVVDVNDNKPDISVNLMSTGNEEIAYISETAPVDTFVALVSVNDLDSGLNGEVECRLYGQGHFRLQKSYEKNYMILTNVTL

ZPcdh19I2 383 DREQRDMYNLTILAEDSGYPPLRSSKSFAVKVTDENDNPPYFTKPHYQAMVLENNVPGAFLLAVSARDPDLGMNGTVSYEIIKSEVRGMSVESYVTVN HPCDH19V2 385 DREQHDQYNLTIOARDGGVPMLOSAKSFTVLITDENDNHPHFSKPYYQVIVQENNTPGAYLLSVSARDPDLGLNGSVSYOIVPSQVRDMPVFTYVS IN MPcdh19V2 385 DREQHDQYNLTI QARDSGVPMLQSAKSFTVRITDENDNHPHFSKPYYQVIVQENNT PGAYLLSVSARDPDMGLNGSVSYQIVPSQVRDMPVFTYVSIN

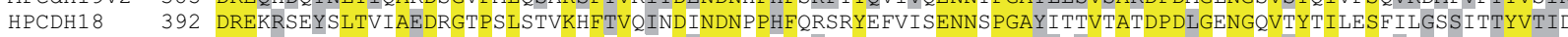
ZPcdh18a 392 DREKRSEFSLTVIAEDKGSPSLSTIKNFIVEVQDENDNAPSFAKSRYEISKAENNSPGAYLSSVKASDPDLGPNGQVSYSILESMVHGSSISTYVTID

ZPcdh19I2 481 SN-GEIYGVRAFNHEDTRTFEFKVSAKDGGDP-PLTSNATVRIVVLDVNDNTPVMTTPPLVNGTAEVSIPKNAGVGYLVTQIKADDYDEGENGRLTYS HPCDH19V2 483 PNSGDIYALRSFNHEQTKAFEFKVLAKDGGLP-SLQSNATVRVI ILDVNDNTPVITAPPLINGTAEVYI PRNSGIGYLVTVVKAEDYDEGENGRVTYD MPcdh19V2 483 PNSGDIYALRSFNHEQTKAFEFKVLAKDGGLP-SLQSNATVRVIILDVNDNTPVITAPPLINGTAEVYI PRNSGIGYLVTVVKADDYDEGENGRVTYD HPCDH18 490 PSNGAIYALRIFDHEEVSQI T FVVEARDGGSPKQLVSNTTVVLTIIDENDNVPVVIGPALRNNTAEITI PKGAESGFHVTRIRAIDRDSGVNAELSCA ZPcdh18a 490 PSNGDIYALRTFDREDVSQISELVQARDSGNP-PLRSNVTVVLTVLDENDNRPVIMMPQLWNHTADVPVSKYAEIGDVVTVVRAMDHDAGANGDLSCS

ZPcdh19I2 577 ISEG-DMAYFEIDQINGEVRTTKTFGENAKPSYQITVVAHDHGQTSLSASAYIVIYLSPDLNAQEQ---------IGPVNLSLIFIIALGSIAVILFV HPCDH19V2 580 MTEG-DRGFFEIDQVNGEVRTTRTFGESSKSSYELIVVAHDHGKTSLSASALVLIYLSPALDAQES----------MGSVNLSLIFI IALGS IAGILFV MPcdh19V2 580 MTEG-DRGFFEIDQVNGEVRTTRTFNENSKPSYEL IVVAHDHGKTSLSASALVLIYLSPALDAQES---------MGSVNLSLI I I IALGS IAGILFV HPCDH18 588 IVAGNEENIFIIDPRSCDI HTNVSMDSVPYTEWELSVI IQDKGNPQLHTKVLLKCMIFEYAESVTSTAMTS--VSQASLDVSMIII ISLGAICAVLIV ZPcdh18a 587 VVGGNEAGYFNMDPKTCEIRTNVSIQEVPQDHVELTILVQDHGTPTLSARALLRLSLYENIENLMNPHLTGGRNGDGPLDVSMIIIISLGAICAVLLI

ZPcdh19I2 665 TMIFVAVKCKRDNKEIRTYNCRVAEYSYGNQKKSSKKKKLSKNDIRLVPRDVEETDKMNVVSCSSLTSSLNYFDYHQQTLPLGCRRSESTFLNVENQN HPCDH19V2 668 TMIFVAIKCKRDNKEIRTYNCRIAEYSYGHQKKSSKKKKISKNDIRLVPRDVEETDKMNVVSCSSLTSSLNYFDYHQQTLPLGCRRSESTFLNVENQN MPcdh19v2 668 TMIFVAIKCKRDNKEIRTYNCRIAEYSYGHQKKSSKKKKISKNDIRLVPRDVEETDKMNVVSCSSLTSSLNYFDYHQQTLPLGCRRSESTFLNVENQN HPCDH18 684 IMVLFATRCNREKKDTRSYNCRVAESTYQHHPKRPSRQ-IHKGDITLVP-TINGTLPIRSHHRSSPSSSP-----TLERGQMGSRQS HNSHQSLNSLV ZPcdh18a 685 IMVAFALRCSREKKDTRSYNCRVAESTYQQHPKKPSRQ-IHKGDITLMP-TVNGTLPIRAHHR-SPTSSP------GAERAHMGSRQSQHSRQSLNSLV

$$
\mid<\mathrm{CM} 1 \longrightarrow
$$

ZPcdh19I2 763 SRNAAPNHGYHHTFTGQGPQQPDLI INGMPLPETENYSI DSSYVNSRAHLIKSTSTFKDMEGNSLKDSGHEESDQTDSEHDVQRGHYADTAVNDVLNM HPCDH19V2 766 TRNTSANHI YHHSENSQGPQQPDLI INGVPLPETENYSF DSNYVNSRAHLIKS-STFKDLEGNSLKDSGHEESDQTDSEHDVQRSLYCDTAVNDVLNT MPcdh19V2 766 TRNTTASHIYHHSENSQGPQQPDLI INGVPLPETENYSFDSNYVNSRAHLIKS-STFKDLEGNSLKDSGHEESDQTDSEHDVQRSLYCDTAVNDVLNT HPCDH18 775 TISSNHVPENFSLELTHATPAVEQVSQLLSMLHQGQYQPRPSFRGNKYSRSYR-YALQDMDKFSLKDSGRGDSEAGDSDYDLGRDSPIDRLLGEGFSD ZPcdh18a 775 TISSNHIPENFALELTHATPPVE----------GQYQPRPSERGNKYSRSYR-YALQDMDKFSLKDSGRGDSDAGDSDCEMGRDSPIDRLLGDGEGD

$$
\underset{H C O D E C R I L G H S D R C W M P}{\longleftrightarrow}
$$
$\begin{array}{lll}\text { HPCDH19V2 } & 863 & \text { SVTSMGSQMPDHDQNEGFHCREECRILGHSDRCWMP } \\ \text { MPCdh19V2 } & 863 & \text { SVTSMGSQMPDHDQNEGFHCREECRILGHSDRCWMP }\end{array}$ HPCDH18 872 LFLTDG--RIPAAMR---LCTEECRVLGHSDQCWMP ZPcdh18a 861 LFHSDGHHRLHPVMR---LCTEECRVLGHSDQCWMP

\section{B}

\begin{tabular}{|l|l|l|l|l|l|l|l|l|}
\hline & ZPcdh1912 & HPCDH19V2 & MPcdh19V2 & HPCDH18 & MPcdh18 & ZPcdh18a & HPCDHV2 & ZPcdh1 \\
\hline ZPcdh1912 & & $71.28 \%$ & $71.76 \%$ & $34.20 \%$ & $34.29 \%$ & $35.84 \%$ & $22.75 \%$ & $22.05 \%$ \\
\hline HPCDH19V2 & & & $96.46 \%$ & $33.99 \%$ & $32.80 \%$ & $35.56 \%$ & $22.46 \%$ & $22.29 \%$ \\
\hline MPcdh19V2 & & & & $33.57 \%$ & $32.95 \%$ & $35.78 \%$ & $22.79 \%$ & $23.30 \%$ \\
\hline HPCDH18 & & & & & $93.12 \%$ & $66.84 \%$ & $22.06 \%$ & $22.95 \%$ \\
\hline MPcdh18 & & & & & & $66.10 \%$ & $22.31 \%$ & $23.60 \%$ \\
\hline ZPcdh18a & & & & & & & $23.71 \%$ & $23.41 \%$ \\
\hline HPCDH1V2 & & & & & & & & $68.64 \%$ \\
\hline ZPcdh1 & & & & & & & & \\
\hline
\end{tabular}

ZPCdh19I2 861 TVPSNNSQIPDQDQSEGFHCQDECRILGHSDRCWM

Fig. 3. Amino acid sequence alignment and pairwise comparisons of several protocadherins. (A) Amino acid sequence alignment between the deduced zebrafish protocadherin-19 isoform 2 amino acid sequence, human protocadherin-19 variant 2 , mouse protocadherin-19 variant 2 , and two $82-$ protocadherins: human protocadherin-18 and zebrafish protocadherin-18a. The alignment shows sequences between published sequences from the EC1 to the end of the conserved cytoplasmic motif 2. Sequences highlighted by yellow boxes indicate residues that are common to all five sequences, and sequences highlighted by gray boxes indicate amino acids that are identical to at least three of the sequences. The conserved cytoplasmic motifs CM1 and CM2 are indicated. (B) Sequence identity percentages for pairwise comparisons between the above five $\delta 2$-protocadherin sequences, a mouse protocadherin-18 sequence (MPcdh18), and two $\delta 1$-protocadherin sequences: human protocadherin-1 variant 2 and zebrafish protocadherin-1. Diagonal shaded boxes indicate sequence comparisons between the same sequences, and therefore, represent 100\% identity. Sequence comparisons were performed using ClustalW2. Other abbreviations are the same as in Fig. 2. 


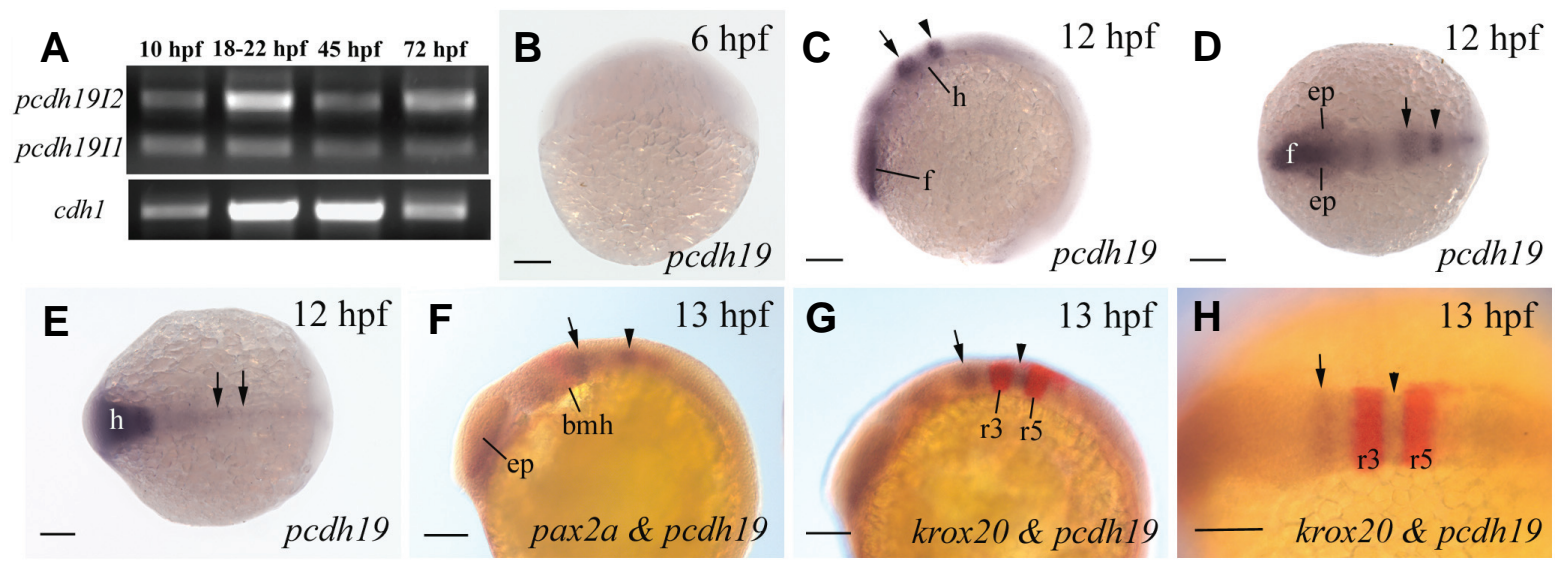

Fig. 4. Pcdh19 expression in 6-13 hpf zebrafish embryos. (A) RT-PCR analysis of Pcdh19 isoforms 1 and 2 expression in embryonic zebrafish using total RNAs. RT-PCR for cdh1 was performed as loading control. The remaining panels show whole mount embryos labeled with Pcdh19 cRNA probes (B-E), Pcdh19 and pax2a cRNA probes (F), or Pcdh19 and krox20 cRNA probes (G,H). (B,C) Lateral views of the entire embryos (head towards the lower left corner for C). (D,E) Dorsal views (anterior to the left) of the entire embryos. (F,G) Lateral views of the anterior half of the embryos (anterior to the left and dorsal up), while (H) is a dorsal view of the presumptive hindbrain region of an embryo (anterior to the left). The arrow and arrowhead in (C, D, F, G and H) point to the first and second Pcdh19 expression domains, respectively, in the presumptive hindbrain. The two arrows in panel E indicate Pcdh19 expression in the middle neural keel. Abbreviations: bmh, boundary of the mid-and hindbrains; ep, eye premordium; $f$, presumptive forebrain; $h$, presumptive hindbrain; $r 3$ and r5, rhombomeres 3 and 5, respectively. Scale bars, $100 \mu \mathrm{m}$.
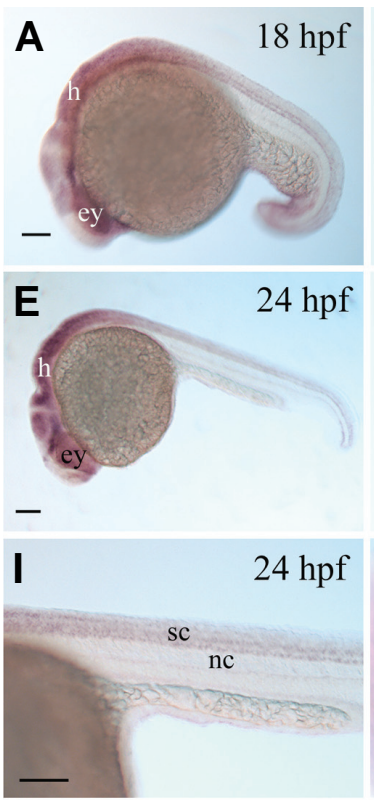

$\mathbf{M}$

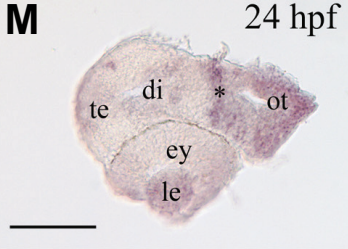

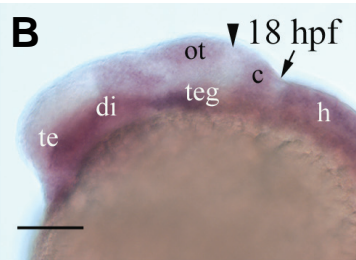
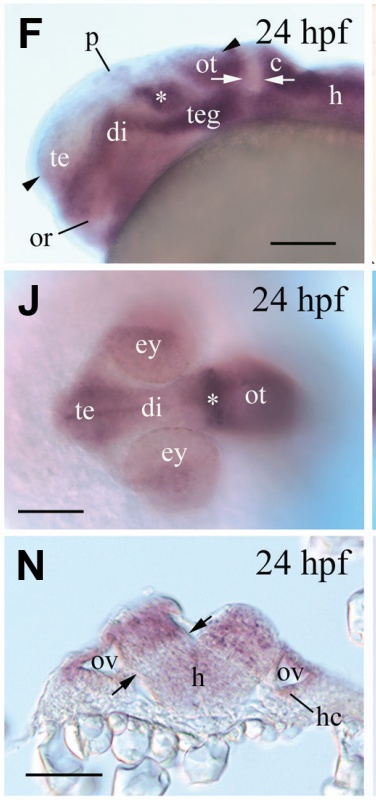
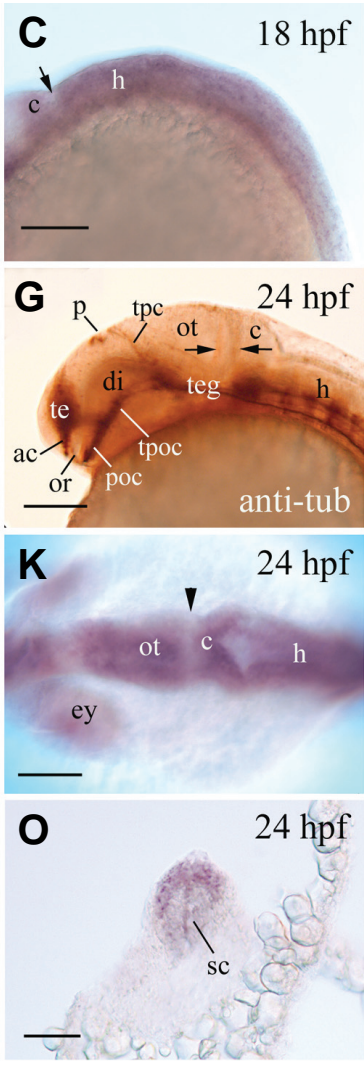
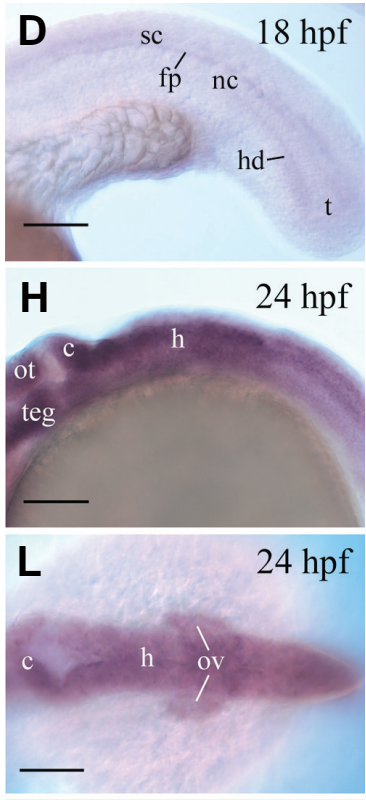

$\mathbf{P}$

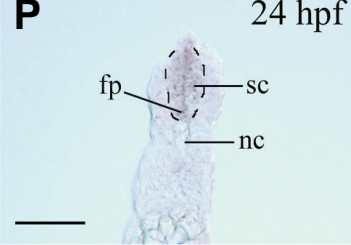

Fig. 5. Pcdh19 expression in 18 and 24 hpf zebrafish embryos. (A-F) and (H-L) Whole mount embryos labeled with Pcdh19 cRNA. (G) is the head region of a whole mount embryo labeled with the anti-acytelated tubulin antibody. $(\mathbf{A}, \mathbf{E})$ Lateral views of the entire embryos (head towards the lower left corner). (B,F) Lateral views of the fore-and midbrains, while $\mathbf{( C , H )}$ are lateral views of the hindbrain (anterior to the left, dorsal up). (J,K,L) Dorsal views of the head region (anterior to the left). (D,I) Lateral views of the posterior half of the body and midtrunk region, respectively (anterior to the left). The arrowhead in (B, K), and the opposing arrows in $(\mathbf{F}, \mathbf{G})$ indicate the same region with reduced Pcdh19 expression, located between the posterior border of the optic tectum and boundary of the midhindbrains. The arrow in $(\mathbf{B}, \mathbf{C})$ point to the same area with

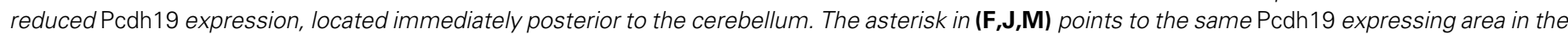

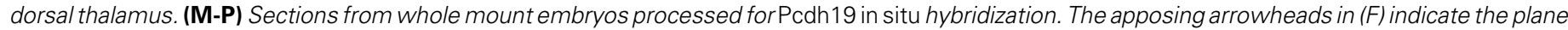

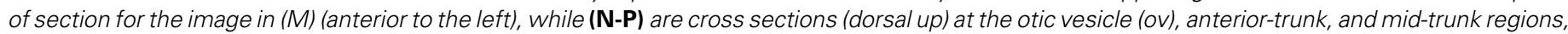

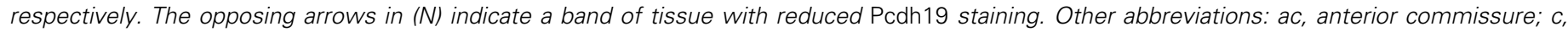

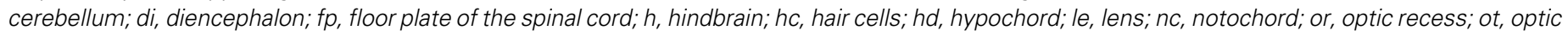

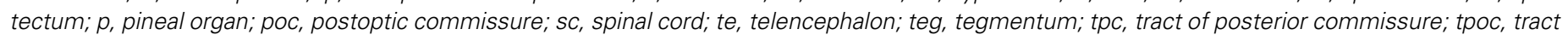
of postoptic commissure. The remaining abbreviations are the same as in Fig. 4 . Scale bars, $100 \mu \mathrm{m}$ for (A-M), and $50 \mu \mathrm{m}$ for (N-P). 


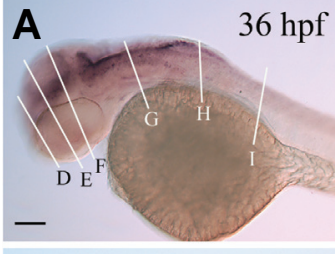

D
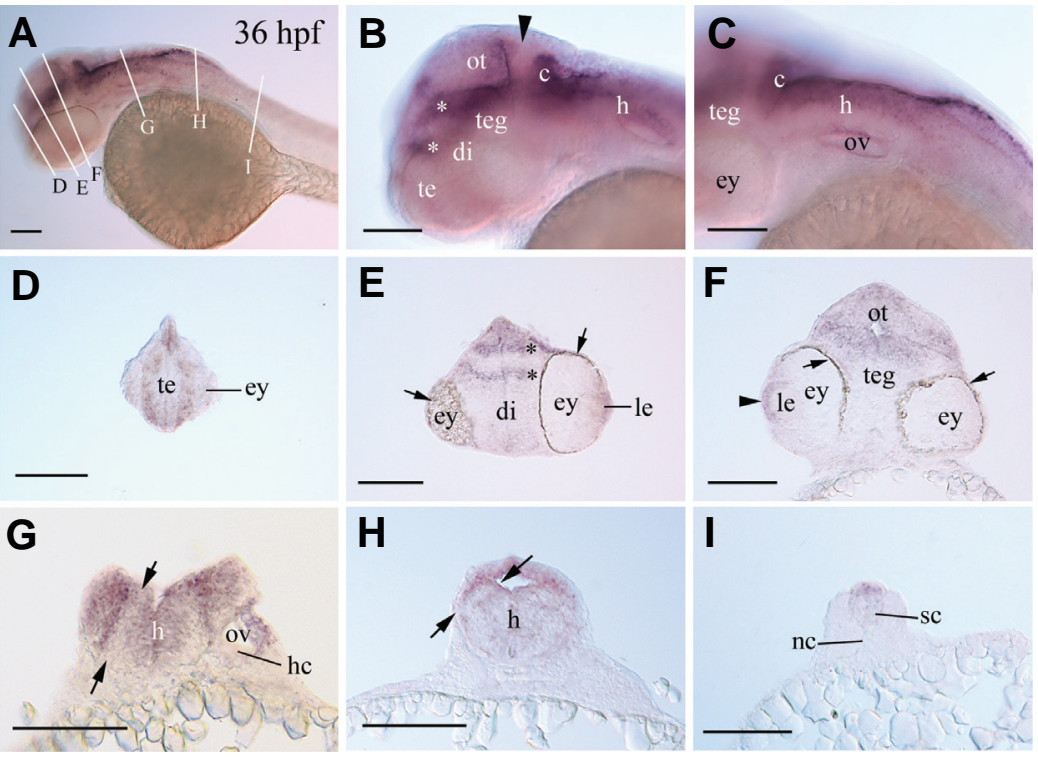

E
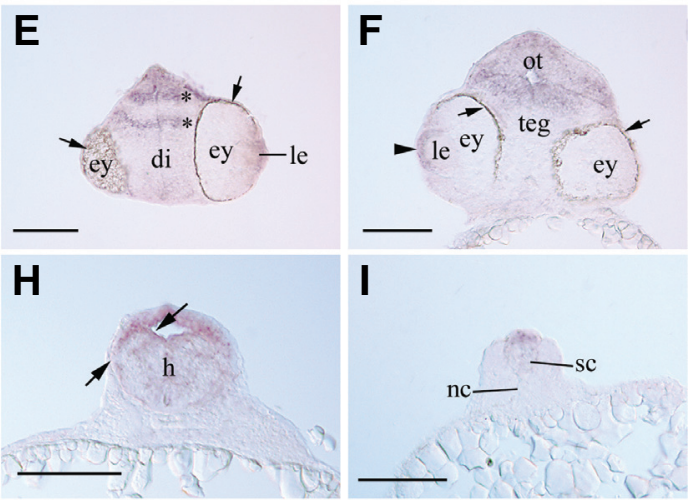

Fig. 6. Pcdh19 expression in $\mathbf{3 6}$ hpf embryos. (A,B,C) Lateral views of whole mount embryos showing the anterior $2 / 3$ of the fish, the anterior head, and posterior head regions, respectively (anterior to the left and dorsal up). (D-I) Cross sections (dorsal up) from whole mount embryos processed for Pcdh19 in situ hybridization. Levels of the sections are shown in (A). The arrowhead in (B) points to the region with reduced Pcdh19 expression (also see Fig. 5F). Arrows in (E,F) indicate pigmented epithelium. The arrowhead in (F) points to Pcdh19 expression in the epithelial layer of the lens. Asterisks in $(B, E)$ indicate the same thalamic regions with stronger Pcdh19 expression. The opposing arrows in $(G, H)$ indicate the band of tissue with reduced Pcdh19 expression (also see Fig. 5N). Abbreviations are the same as in Fig. 5. Scale bars, $100 \mu \mathrm{m}$.

nervous tissue, Pcdh19was also seen in the hypochord below the notochord near the tail (Fig. 5D). Embryos of 24 hpf showed somewhat similar Pcdh19 expression patterns in the fore- and midbrains as $18 \mathrm{hpf}$ embryos, except that the staining was stronger in the dorsal thalamus (ventroanteral to the optic tectum, and adjacent to the tract of the posterior commissure, indicated by an asterisk in Fig. 5F) and optic tectum (Fig. 5F) than $18 \mathrm{hpf}$ embryos (Fig. 5B). In the telencephalon, the Pcdh19 expressing domain was located anterodorsal to the optic recess (Fig. 5 F,G). The stronger labeled regions in the telencephalon, central thalamus and tegmentum appeared to form a continuous thick bank viewing laterally (Fig. 5F). The stripe of tissue between the posterior border of the optic tectum and the boundary of the mid- and hindbrains continued to show much reduced staining (Fig. 5 F,G, indicated by two opposing arrows). In the hindbrain, Pcdh19expression was continuous from the cerebellum to the spinal cord, with stronger expression levels detected in the cerebellum and dorsolateral hindbrain (Fig. $5 \mathrm{H}, \mathrm{N}$ ). A band of tissue between the dorsolateral and the ventromedial hindbrain showed reduced staining (indicated by two opposing arrows in Fig. $5 \mathrm{~N}$ ). In the anterior spinal cord, its dorsal $1 / 3$ area was also more strongly labeled than the ventral spinal cord (Fig. 50), while in the mid-trunk and tail regions of the spinal cord, regional differences in the staining was not detected and the floor plate region continued to express Pcdh19 (Fig. 5P). Pcdh19 was also expressed by the retina, lens (Fig. $5 \mathrm{~J}, \mathrm{M}$ ), and otic vesicle $(5 L$ and $N)$. The lens and peripheral retina (future retinal marginal zones) were more strongly labeled than the central retina (Fig. 5M). Epithelial cells in the lateral otic vesicle and the hair cells were strongly labeled (Fig. 5N).

Pcdh19expression was reduced and regional differences in expression levels became more pronounced in some regions of the CNS in 36 hpf embryos (Fig. 6). Stronger Pcdh19 staining continued to be observed in the dorsal and central thalamus (Fig. 6 B,E,F), the cerebellum (Fig. 6 B,C), and the dorsolateral regions of the hindbrain (Fig. 6 B,C,G,H). In the telecephalon, stronger Pcdh19 expression was found in the lateral portion (Fig. 6D). In the diencephalon, the strongly labeled Pcdh19 expression domains in the dorsal and central thalamus

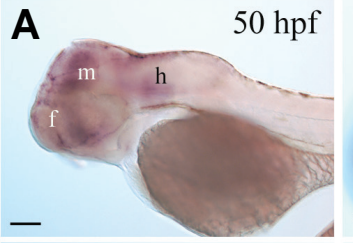

E
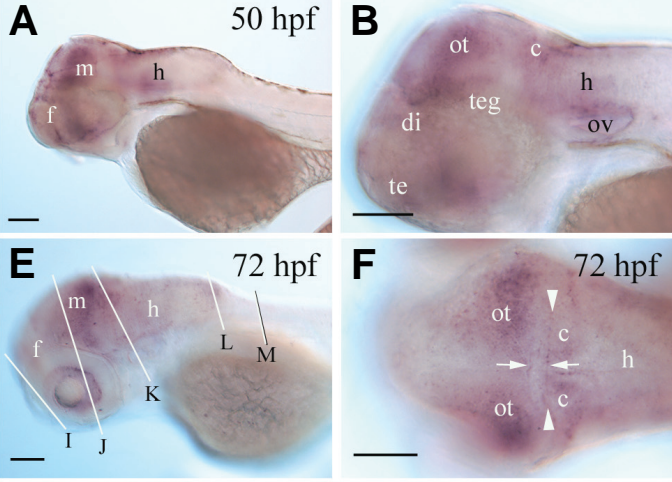

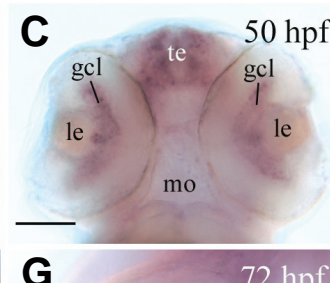

G
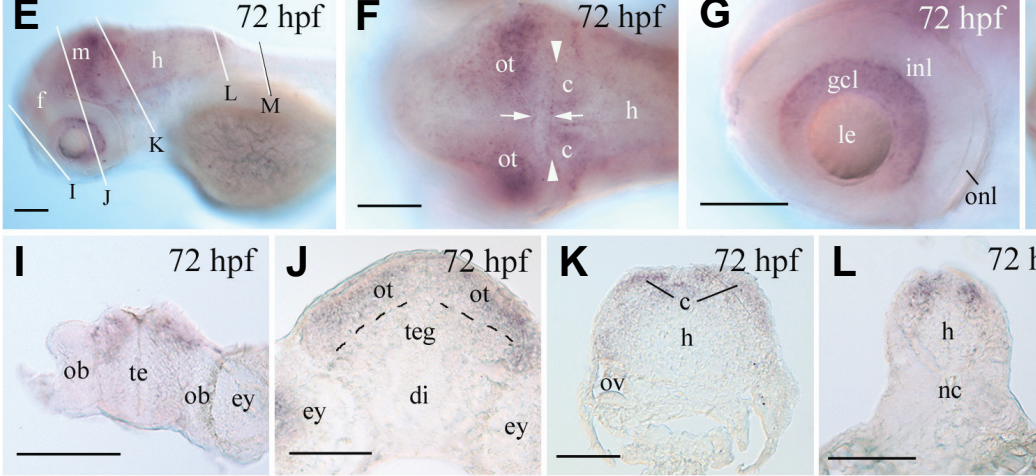
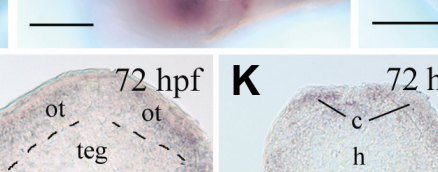

ov

ey
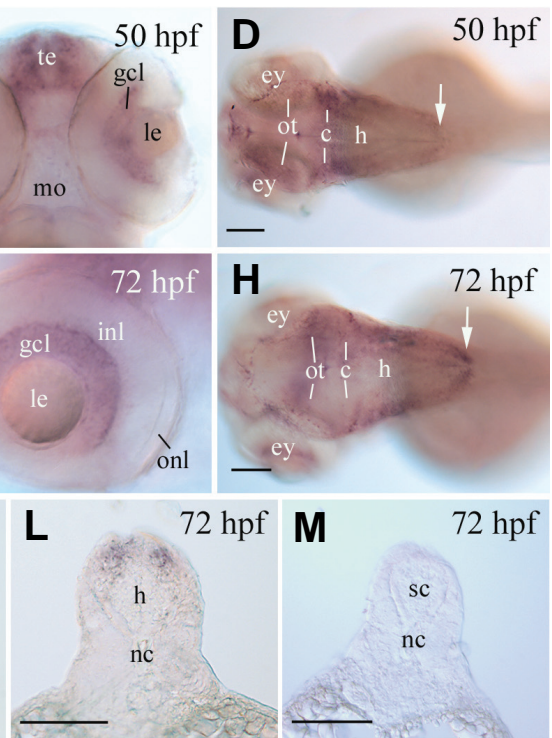

$50 \mathrm{hpf}$

$72 \mathrm{hpf}$

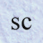

nc

their section levels indicated in (E). The dashed lines in (J) indicate the boundary between the optic tectum and tegmentum. Abbreviations: $f$, forebrain; gcl, retinal ganglion cell layer; inl, inner nuclear layer; m, midbrain; onl, outer nuclear layer. Other abbreviations are as in Fig. 4. Scale bars, 100 um. 
had the appearance of two horizontal bands in cross sections (Fig. $6 \mathrm{E}$, indicated by two asterisks). In the midbrain, Pcdh 19 expression was confined mainly to the optic tectum and dorsal tegmentum (Fig. 6F). Again, the region between the posterior border of the optic tectum and the boundaries of mid-and hindbrains (arrowhead in Fig. 6B), and the region between the dorsolateral and the ventromedial hindbrain (opposing arrows in Fig. $6 \mathrm{G}, \mathrm{H}$ ) had less Pcdh19expression than their neighboring areas. The dorsal spinal cord contained higher Pcdh19 expressing cells than the ventral spinal cord even in the mid-trunk region (Fig. 6I). Pcdh19expression in the retina and lens was reduced compared to $24 \mathrm{hpf}$ embryos, except in the retinal marginal zones and the lens epithelial layer (Fig. 6 E,F). Strong Pcdh19expression continued in the lateral epithelial cells of the otic vesicle, but its expression was reduced in the hair cells (Fig. $6 \mathrm{G}$ ).

Generally speaking, Pcdh19expression in 50 hpf embryos was similar to those of $72 \mathrm{hpf}$ (Fig. 7). Similar to the younger embryos, stronger Pcdh19 staining was seen in the dorsal thalamus and dorsal tegmentum (Fig. 7B), optic tectum (Fig. 7 B,F,J), cerebellum (Fig. 7 B,K), and dorsolateral hindbrain (Fig. 7 D,H,K), but Pcdh19 expression in the central thalamus, tegmentum and hindbrain was reduced compared to 24 and 36 hpf embryos. At $50 \mathrm{hpf}$, Pcdh 19 expression in the telencephalon (Fig. 7C) was mainly in the lateral regions (similar to $36 \mathrm{hpf}$ embryos, Fig. 6D), but stronger expression became restricted to the dorsal telencephalon by $72 \mathrm{hpf}$ (Fig. 7I). The region between the posterior border of the optic tectum and the boundary of the mid-hindbrains continued to show reduced staining (Fig. 7F), while Pcdh19 expression in the ventromedial hindbrain was greatly reduced (Fig. 7K) compared to younger embryos (Figs. 5N and 6G). Pcdh19expression in the spinal cord was no longer detectable at both $50 \mathrm{hpf}$ (Fig. 7D, data not shown for spinal cord sections) and $72 \mathrm{hpf}$ (Fig. $7 \mathrm{H}, \mathrm{M})$. In the eye, Pcdh 19 expression became mainly confined to the retinal ganglion cell layer, while there was no Pcdh19 expression in the lens (Fig. 7 C,G). Pcdh19 expression in the otic vesicle became further reduced (Fig. 7K).

Pcdh19 expression in the embryonic zebrafish is somewhat similar to that in embryonic mice (Gaitan and Bouchard, 2006). In both species, early Pcdh19 expression is mainly confined to the nervous system. Pcdh19 is found in the telencephalon and diencephalon, in the spinal cord, in the developing retinal ganglion cell layer and lens. In adult rat brain, Pcdh19was expressed in specific regions of the telencephalon, diencephalon, midbrain and hindbrain (Kim et al. 2007). In addition to the neural tissue, Pcdh19 is also expressed by several other tissues in human (RT-PCR analysis, Wolverton and Lalande 2001) and mouse (Gaitan and Bouchard 2006), including the heart and kidney. It remains to be determined if Pcdh19 is expressed by these tissues in larval and adult zebrafish.

\section{Materials and Methods}

Zebrafish embryos were obtained from in house breeding, and maintained as described in the Zebrafish Book (Westerfield 2005). Embryos for whole mount in situhybridization were raised in PTU (1-phenyl-2-thiourea, $0.003 \%$ ) at $28.5^{\circ} \mathrm{C}$, staged in hours post fertilization, and fixed in phosphate buffered $4 \%$ paraformaldehyde.

\section{Cloning of zebrafish Pcdh19}

A TBlastn search using human $\mathrm{PCDH} 19$ protein sequence as a query resulted in a zebrafish genomic DNA sequence CR318607. A zebrafish Pcdh19cDNA fragment was amplified by PCR from a zebrafish embryonic cDNA Uni-ZAP XR library (discontinued; Stratagene, La Jolla, CA) using primers (forward primer 0, 5'-CGTTAGTCATAGACCTGAACGCCACTGA CC -3, reverse primer 0, 5'-TTACTACCAAGCCACGATGACAGTCTGAGC -3') flanking a predicted zebrafish Pcdh19coding region. The PCR product was cloned in the pCR-Blunt II-TOPO vector (Invitrogen, Carlsbad, CA). Sequences of the clones indicated 2 isoforms, Pcdh 19 isoforms 1 and 2 (GenBank, accession number: AB 362378 and AB 362379, respectively, F. Kubota and T. Murakami). Analysis of the deduced amino acid sequences of these isoforms revealed that they lack the N-terminal 1/3 (from signal sequence to about half of EC3) of the proteins compared to other protocadherins. A BLAST search using the Pcdh19 isoform 2 sequence resulted in a zebrafish cDNA clone sequence (wu:fc83e05, GenBank accession number: BC129243, Strausberg et al.) that contains all the incomplete Pcdh19 isoform 2 sequence, plus most of the missing $\mathrm{N}$ terminal sequence. Compared to other protocadherins including the mammalian Pcdh19 proteins, this zebrafish sequence (zebrafish protocadherin19 isoform 2) appeared to be missing some of the signal sequence in the 5' region. Using primers designed according to genomic sequence of wu:fc83e05 (GenBank accession number: NW 001884478) and mRNA sequence of the wu:fc83e05 (forward primer 1, 5'. CGCGTGAAGACAGACATCAA-3', forward primer 2, 5'TTCCAAGGACATGGATTTCG-3', 58 and 25 nucleotides, respectively, 5 to the starting codon of the published wu:fc $83 \mathrm{e} 05$ sequence; reverse primer 1, 5'-AGTAGACCACCTCGCCATTG-3', corresponding to the nucleotides 748 to 767 of the wu:fc8305), and total RNAs from 24-70 hpf whole zebrafish embryos, we performed RT-PCR and obtained the missing signal sequence (Fig. 1)

\section{RT-PCR analysis of Pcdh19 expression in developing zebrafish}

RT-PCR analysis of Pcdh19 isoforms temporal expression profiles was performed using Pcdh19 specific primers (forward primer 3, 5'GCCTTGGGCTCTATTGCAGTCA-3'; reverse primer 2, 5'AGCATAGTGCCCTCTCTGGA-3', corresponding to nucleotides 12841305 and 1855-1874, respectively, of zebrafish Pcdh19isoform2, GenBank accession number AB 362379). These primers amplified bands of $377 \mathrm{bp}$ and 590 bp for Pcdh19isoforms 1 and 2, respectively (Fig. 4A). Zebrafish cdh1transcripts (Liu et al. 2007) were used as the control for the RT-PCR experiments because $c d h$ 1 was shown to be strongly expressed by young zebrafish embryos (Babb et al. 2005).

\section{In situ hybridization and immunocytochemistry}

For obtaining a Pcdh19DNA fragment (corresponding to the nucleotides 43-985 of the incomplete zebrafish Pcdh19isoforms 1 and 2) as a template for synthesizing cRNA probes, zebrafish Pcdh19 specific primers (forward primer 4, 5'-CAATGGCGAGGTGGTCTACT-3'; reverse primer 3, 5'-CAACTCCAGCGTTTTTAGGG-3'), and total RNA isolated from 20$50 \mathrm{hpf}$ whole zebrafish embryos were used for RT-PCR. This cDNA fragment was cloned into the PCRII-TOPO vector (Invitrogen), and was verified by restriction mapping and a PCR experiment using a pair of $P c d h 19$ specific primers that were internal to the previous set of primers (forward primer 5, 5'-GCCCGAAATCAAACTGTTGT-3'; reverse primer 4, 5'-GCACCTCCGATTTGATGATT-3'). This experiment produced a cDNA fragment corresponding to the nucleotides 266-729 of the incomplete zebrafish Pcdh19 isoforms 1 and 2). The larger Pcdh19 cDNA fragment in the pCRII-TOPO vector was used as a template for the synthesis of digoxigenin-labeled zebrafish Pcdh19 RNA sense or antisense probes for in situ hybridization. Both Pcdh19 isoforms contained this cDNA fragment. cDNAs used to generate the fluorescein-labeled antisense pax2a and krox20 were provided by Drs. Pamela Raymond (University of Michigan) and Lisa Maves (Fred Hutchinson Cancer Research Center), respectively. Detailed procedures for the cRNA probe synthesis and whole mount in situ hybridization were described previously (Liu et al. 1999; Liu et al. 2007; Kubota et al. 2008). There was no 
staining in zebrafish embryos from 24-50 hpf using the sense Pcdh19 probes (data not shown).

Anti-acetylated tubulin antibody (Sigma, St Louis, MO) was used at 1:3000. The secondary antibody (used at 1:250) was biotinylated antimouse IgG (Vector laboratories, Burlingame, CA). Whole-mount immunocytochemistry was carried out according to protocols described in the Zebrafish Book (Westerfield 2005).

\section{Acknowledgements}

This work was supported by grants from NIHEY13879 to Q. L., Grants in-Aid awarded to T.M. from the Ministry of Education, Culture, Sports, Science, and Technology of Japan (MEXT), and the Initiative for Attractive Education in Graduate Schools awarded to Gunma University from MEXT. We thank Drs. Pamela Raymond (University of Michigan, Ann Arbor, MI) and Lisa Maves (Fred Hutchinson Cancer Research Center, Seattle, WA) for the pax2a andkrox20 containing plasmids, respectively.

\section{References}

BABB S.G., BARNETT J., DOEDENS A.L., COBB N., LIU Q., SORKIN B.C., YELICK P.C., RAYMOND P.A. and MARRS J.A. (2001). Zebrafish E-cadherin: expression during early embryogenesis and regulation during brain development. Dev Dyn 221: 231-237.

FRANK M. and KEMLER R. (2002). Protocadherins. Curr Opin Cel/ Bio/14: 557562.

GAITAN Y. and BOUCHARD M. (2006). Expression of the $\delta$-protocadherin gene Pcdh19 in the developing mouse embryo. Gene Expr Patterns 6: 893-899.

GUMBINER B.M. (2005). Regulation of cadherin-mediated adhesion in morphogenesis. Nat Rev Mol Cell Bio/6: 622-634.

KIM S.-Y, SUN CHUNG H, SUN W. and KIM H. (2007). Spatiotemporal expression pattern of non-clustered protocadherin family members in the developing rat brain. Neurosci 147: 996-1021.

KRAUSS S, JOHANSEN T, KORZH V, and FJOSE A. (1991). Expression of the zebrfish paired box gene pax[zf-b] during early neurogenesis. Development 113: 1193-1206.
KUBOTA F, MURAKAMI T, TAJIKA Y, and YORIFUJI H. (2008). Expression of protocadherin-18 in the CNS and pharyngeal arches of zebrafish embryos. Int $J$ Dev Bio/52: 397-405

LIU Q, SANBORN K.L, COBB N, RAYMOND P.A, and MARRS J.A. (1999). Rcadherin expression in the developing and adult zebrafish visual system. $J$ Comp Neuro/410: 303-319.

LIU B, DUFF R.J, LONDRAVILLE R.L, MARRS J.A, and LIU Q. (2007). Cloning and expression analysis of cadherin7 in the central nervous system of the embryonic zebrafish. Gene Expr Patterns 7: 15-22.

NOONAN J.P, GRIMWOOD J, SCHMUTZ J, DICKSON M, and MYERS R.M. (2004). Gene conversion and the evolution of protocadherin gene cluster diversity. Genome Res 14: 354-366

NOLLET F, KOOLS P, and VAN ROY F. (2000). Phylogenetic analysis of the cadherin superfamily allows identification of six major subfamilies besides several solitary members. J Mol Bio/299: 551-572.

OXTOBY L, and JOWETT T. (1993). Cloning of the zebrafish krox-20 (krx-20) and its expression during hindbrain development. Nucleic Acids Res21: 1087-1095.

REDIES C, VANHALST K, and VAN ROY F. (2005). $\delta$-Protocadherins: unique structures and functions. Cel/ Mol Life Sci62: 2840-2852.

SUZUKI S.T. (1996). Protocadherins and diversity of the cadherin superfamily. $J$ Cell Sci 109: 2609-2611.

VAN ROY F, and BERX G (2008). The cell-cell adhesion molecule E-cadherin. Cell Mol Life Sci65: 3756-3788.

VANHALST K, KOOLS P, STATES K, VAN ROY F. and REDIES C. (2005). $\delta$ Protocadherins: a gene family expressed differentially in the mouse brain. Cell Mol Life Sci62: 1247-1259.

WESTERFIELD M. (2005). The zebrafish Book. Eugene, OR: University of Oregon Press.

WOLVERTON T, and LALANDE M. (2001). Identification and characterization of three members of a novel subclass of protocadherins. Genomics 76: 66-72.

WU Q. (2005). Comparative genomics and diversifying selection of the clustered vertebrate genes. Genetics 169: 2179-2188.

YAGI T. and TAKEICHI M. (2000). Cadherin superfamily genes: functions, genomic organization, and neurologic diversity. Genes Dev14: 1169-1180.

\section{Further Related Reading, published previously in the Int. J. Dev. Biol.}

See Special Issue Pattern Formation edited by Michael K. Richardson and Cheng-Ming Chuong at:

http://www.ijdb.ehu.es/web/contents.php?vol=53\&issue=5-6

Expression of protocadherin 18 in the CNS and pharyngeal arches of zebrafish embryos Fumitaka Kubota, Tohru Murakami, Yuki Tajika and Hiroshi Yorifuji Int. J. Dev. Biol. (2008) 52: 397-405

Cadherin-6 is required for zebrafish nephrogenesis during early development Fumitaka Kubota, Tohru Murakami, Kenji Mogi and Hiroshi Yorifuji Int. J. Dev. Biol. (2007) 51: 123-129

Cadherin-mediated cell-cell adhesion and tissue segregation in relation to malignancy Ramsey A. Foty and Malcolm S. Steinberg Int. J. Dev. Biol. (2004) 48: 397-409

Discovery and characterization of the cadherin family of cell adhesion molecules. An interview with Masatoshi Takeichi Douglas Sipp

Int. J. Dev. Biol. (2004) 48: 387-396

Transcriptional regulation of cadherins during development and carcinogenesis Héctor Peinado, Francisco Portillo and Amparo Cano Int. J. Dev. Biol. (2004) 48: 365-375

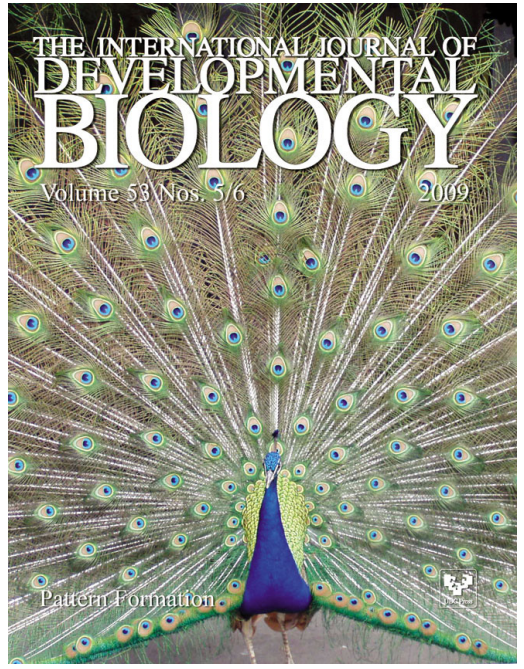

